\title{
Anomaly detection optimization using big data and deep learning to reduce false-positive
}

\author{
Khloud Al Jallad ${ }^{1 *}$, Mohamad Aljnidi ${ }^{1}$ and Mohammad Said Desouki ${ }^{2}$
}

\author{
${ }^{*}$ Correspondence: \\ khloud.aljallad@hiast.edu.sy \\ ${ }^{1}$ Faculty of Information \\ Technology, Higher Institute \\ for Applied Sciences \\ and Technology, Damascus, \\ Syria \\ Full list of author information \\ is available at the end of the \\ article
}

\begin{abstract}
Anomaly-based Intrusion Detection System (IDS) has been a hot research topic because of its ability to detect new threats rather than only memorized signatures threats of signature-based IDS. Especially after the availability of advanced technologies that increase the number of hacking tools and increase the risk impact of an attack. The problem of any anomaly-based model is its high false-positive rate. The high false-positive rate is the reason why anomaly IDS is not commonly applied in practice. Because anomaly-based models classify an unseen pattern as a threat where it may be normal but not included in the training dataset. This type of problem is called overfitting where the model is not able to generalize. Optimizing Anomaly-based models by having a big training dataset that includes all possible normal cases may be an optimal solution but could not be applied in practice. Although we can increase the number of training samples to include much more normal cases, still we need a model that has more ability to generalize. In this research paper, we propose applying deep model instead of traditional models because it has more ability to generalize. Thus, we will obtain less false-positive by using big data and deep model. We made a comparison between machine learning and deep learning algorithms in the optimization of anomaly-based IDS by decreasing the false-positive rate. We did an experiment on the NSL-KDD benchmark and compared our results with one of the best used classifiers in traditional learning in IDS optimization. The experiment shows 10\% lower false-positive by using deep learning instead of traditional learning.
\end{abstract}

Keywords: Intrusion detection systems (IDS), Security intelligence optimization, Unknown threats, Big data, NSL-KDD dataset, False-positive

\section{Introduction}

Signature-based Intrusion detection systems are not suitable anymore to be used in nowadays network environment. Because signature-based models are not able to detect new threats and unknown attacks. Due to technology improvement, the number of attacks is increasing exponentially. Statistics show that attacks number increases with a rate of $100 \%$ each year causing huge money loss, about tens of millions of dollars for ransomware attacks only. This high number of millions of new threats that are developed every day, reduces the effectiveness of signature-based IDS because it is not a practical solution to update the signatures databases every few minutes. Anomaly-based IDS can be a better alternative of signature-based IDS because it is more suitable for nowadays 
challenges of new threats. Because it can detect new threats but still not practically used because of its high false-positive rate. The reason behind the high false-positive rate is that anomaly model classifies an unseen pattern that did not learn it in normal cases, as an abnormal case. The reason behind high false-positive is that the model has overfitting what means it cannot generalize. The solution may seem easy, by extending the training dataset to include all normal cases. But that still not a practical solution for a long time. Although we can add much more normal cases to datasets, we still need a model with higher ability of generalization. In this paper, we propose using deep learning with big data to solve this problem. Big data allows us to use big datasets for training to reduce the false-positive rate by including much more normal cases. And deep learning needs large datasets for training without facing the overfitting problem as it may have more ability to generalize than traditional learning models. We compare results with one of the best traditional learning used classifiers on the NSL-KDD benchmark dataset. Results show reducing false-positive to lower $10 \%$ than already used classifiers.

The paper is organized as follows, we will talk about related works in "Related Work Section". The proposed method is explained in detail in "Method Section". "Data Section" contains detailed information about used dataset. We will discuss results in "Results and Discussion Section". We will talk about conclusion and future vision in "Conclusion and Future Work Section".

\section{Related work}

To optimize IDS, there are two general resolving directions. First way is to collect more data. Network security data and intrusion data are hard to collect because of data privacy concerns. In addition to the challenges of labeling data, which may be time-consuming for experts to do explanations and labeling process. Second way is studying how to increase the performance on small datasets, which is very important because the insights we can get from such researches can be implemented in big data researches. This paper is considered under the second way. We propose implementing deep learning instead of traditional learning. We choose to compare our works with SVM, shown in Fig. 1. Because SVM is the most popular traditional learning model used in network security and intrusion detection systems along years even in the era of big data. SVM has received a lot of interest in IDS optimization domain because it has proved by lots

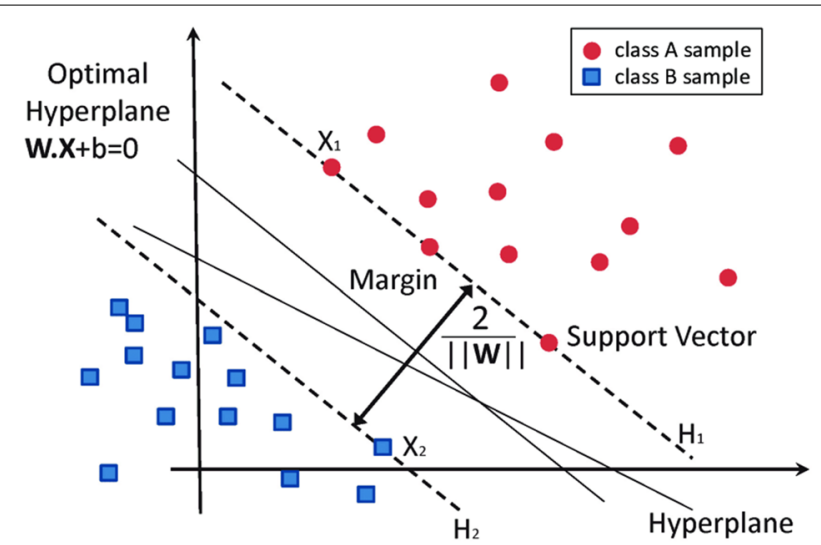

Fig. 1 SVM [6]. Shows the main concept of SVM. Its margins and support vectors 
of experiments that it is one of the best classifiers in anomaly-based intrusion detection systems [1-5]

Find Table 1 from [1], that contains research experiments that used basically SVM and optimized it by adding another model to its result or to its input.

\section{Method}

The problem of any anomaly model is high false-positive rate, because it classifies any unseen pattern as abnormal where it may be a normal pattern but not included in the training set. Having a big training dataset that includes all possible normal cases has been considered as a theoretical idea that could not be applied in practice. Although we can increase the number of training samples to include much more normal cases, still we need a model that has more ability to generalize. We propose deep learning model instead of traditional learning as it may have more ability to generalize.

The proposed solution is to use a big data training set, but also to use a deep learning model to solve overfitting problem that occurs on the traditional learning models.

We have done many experiments [26] that we care about collective and contextual attacks. Where we chose using a deep network with type of recurrent so we can handle sequences of actions or events. We chose LSTM to deal with sequences of inputs and focus on context.

We were curious if LSTM can also achieve better results on small benchmark datasets or not. So we applied this experiment, we do not use sequences or even big data in this experiment. Only small benchmark data is used with LSTM.

\section{Proposed algorithm-long short term memory(LSTM)}

LSTM is type of Deep Learning Recurrent Neural Network (RNN). see Fig. 2. RNN is extension of a convention feed-forward neural network. Unlike feedforward neural networks, RNN have cyclic connections making them powerful for modeling sequences. As a human no one think of each event separately. For example, when you are reading this article you read each word but you understand it in the context of this article, so that you understand the whole concept of this paper. That is the idea of RNN that has a loop to deal with input as a sequence, and that what we need to handle each event on network within its context.

LSTM is a special case of RNN that solves problems faced by the RNN model [27, 28]

1. Long term dependency problem in RNNs.

2. Vanishing Gradient \& Exploding Gradient

LSTM is designed to overcome vanishing gradient descent because it avoids long-term dependency problem. To remember information for long periods of time, each common hidden node is replaced by LSTM cell. Each LSTM cell consists of three main gates such as input gate $i t$, forget gate $f t$, and output gate $o t$. Besides $c t$ is cell state at time $t$.

LSTM architecture is shown in Fig. 3, LSTM cell is shown in Fig. 4.

Figure 5 shows the equations to calculate the values of gates 
Table 1 Comparisons between Researches used SVM as basic Classifier

\begin{tabular}{|c|c|c|c|c|}
\hline Approach & Working idea & Dataset & Detection rate & False alarm \\
\hline $\begin{array}{l}\text { Unsupervised anomaly detec- } \\
\text { tion system [7] }\end{array}$ & $\begin{array}{l}\text { Tune and optimize automati- } \\
\text { cally the values of param- } \\
\text { eters without pre-defining } \\
\text { them }\end{array}$ & $\begin{array}{l}\text { From Kyoto } \\
\text { University } \\
\text { honeypot }\end{array}$ & - & - \\
\hline Multiclass SVM [8] & $\begin{array}{l}\text { Attributes are optimized using } \\
\text { k-fold cross validation. This } \\
\text { technique can be used to } \\
\text { decrease the rate of False- } \\
\text { Negatives in the IDS }\end{array}$ & Self & - & - \\
\hline OC-SVM One-Class SVM [9] & $\begin{array}{l}\text { Multistage OC-SVM and fea- } \\
\text { ture extraction represents a } \\
\text { method to detect unknown } \\
\text { attacks. Method is poor in } \\
\text { second stage classifier to } \\
\text { detection rate of unknown } \\
\text { attacks }\end{array}$ & From Kyoto & 80.00 & 20.94 \\
\hline $\begin{array}{l}\text { IG-ABC-SVM Information Gain- } \\
\text { Artificial Bee Colony [10] }\end{array}$ & $\begin{array}{l}\text { A combining IG feature selec- } \\
\text { tion and SVM classifier in IDS } \\
\text { model is proposed. Experi- } \\
\text { ments using just two swarm } \\
\text { intelligence algorithms }\end{array}$ & NSL-KDD & 98.53 & 0.03 \\
\hline SbSVM [11] & $\begin{array}{l}\text { Autonomous labeling } \\
\text { algorithm of normal traffic } \\
\text { (when the class distribu- } \\
\text { tion is not imbalanced) Not } \\
\text { evaluated for real time case }\end{array}$ & DARPA & 99 & 5.5 \\
\hline $\begin{array}{l}\text { RS-ISVM- reserved set -Incre- } \\
\text { mental SVM [12] }\end{array}$ & $\begin{array}{l}\text { An incremental SVM training } \\
\text { algorithms is used, hybrid } \\
\text { with modifying kernel } \\
\text { function U-RBF Foresee- } \\
\text { ing attacks, specifically for } \\
\text { attacks of U2R and R2L may } \\
\text { not tolerate but oscillation } \\
\text { problem solved }\end{array}$ & KDD Cup 1999 & 89.17 & 4.9 \\
\hline SVM-GA [13] & $\begin{array}{l}\text { Hybrid model by combining } \\
\text { (GA and SVM) }\end{array}$ & KDD CUP 1999 & 98.33 & 0.50 \\
\hline $\begin{array}{l}\text { Genetic principal } \\
\text { Component [14] }\end{array}$ & $\begin{array}{l}\text { Subset selection using GA } \\
\text { and PCA }\end{array}$ & KDD cup 1999 & 99.96 & 0.49 \\
\hline SVM and NN [15] & $\begin{array}{l}\text { Hybrid process Most sig- } \\
\text { nificant performance as far } \\
\text { as training time but time } \\
\text { consuming and hard task } \\
\text { to trigger }\end{array}$ & DARPA & 99.87 & - \\
\hline $\begin{array}{l}\text { N-KPCA-GA-SVM kernel prin- } \\
\text { cipal component analysis- } \\
\text { genetic algorithm (GA)-SVM } \\
{[16]}\end{array}$ & $\begin{array}{l}\text { Hybrid of KPCA, SVM and } \\
\text { GA algorithms. Faster con- } \\
\text { vergence speed. Performs } \\
\text { higher predictive accuracy } \\
\text { and better generalization } \\
\text { But have complex structure } \\
\text { and have latency for real } \\
\text { time application }\end{array}$ & KDD CUP99 & 96.37 & 0.95 \\
\hline $\begin{array}{l}\text { CSV-ISVM } \\
\text { Candidate Support Vector } \\
\text {-Incremental SVM [17] }\end{array}$ & $\begin{array}{l}\text { Candidate Support Vector } \\
\text {-Incremental SVM improves } \\
\text { detection rate and false } \\
\text { alarm rate }\end{array}$ & KDD Cup 1999 & 90.14 & 2.31 \\
\hline $\begin{array}{l}\text { Hybrid approach of } \\
\text { K-Medoids, SVM and Naïve } \\
\text { Bayes [18] }\end{array}$ & $\begin{array}{l}\text { Hybrid learning approach } \\
\text { through a combination } \\
\text { of K-Medoids clustering, } \\
\text { Selecting Feature using } \\
\text { SVM, and Naïve Bayes } \\
\text { classifier }\end{array}$ & KDD Cup 1999 & 90.1 & 6.36 \\
\hline
\end{tabular}


Table 1 (continued)

\begin{tabular}{|c|c|c|c|c|}
\hline Approach & Working idea & Dataset & Detection rate & False alarm \\
\hline $\begin{array}{l}\text { Distance of sum-based SVM } \\
\text { [19] }\end{array}$ & $\begin{array}{l}\text { Hybrid learning method } \\
\text { named distance sum-based } \\
\text { support vector machine } \\
\text { (DSSVM) }\end{array}$ & KDD Cup 1999 & & \\
\hline SVM and GA [20] & $\begin{array}{l}\text { Hybrid method consisting of } \\
\text { GA and SVM for intrusion } \\
\text { detection system }\end{array}$ & KDD Cup 1999 & 0.98 & 0.017 \\
\hline PCA and SVM [21] & $\begin{array}{l}\text { Hybrid model by integrating } \\
\text { the principal component } \\
\text { analysis (PCA) and (SVM) }\end{array}$ & NSL-KDD & 0.9655 & - \\
\hline SVM with GA [5] & $\begin{array}{l}\text { FWP-SVM-genetic algorithm } \\
\text { (feature selection, weight, } \\
\text { and parameter optimization } \\
\text { of support vector machine } \\
\text { based on the genetic } \\
\text { algorithm) }\end{array}$ & KDD Cup 1999 & 96.61 & 3.39 \\
\hline $\begin{array}{l}\text { SVM for Anomaly in smart city } \\
\text { wireless network [22] }\end{array}$ & $\begin{array}{l}\text { Compare SVM and isolation } \\
\text { forests to detect anoma- } \\
\text { lies in a laboratory that } \\
\text { reproduces a real smart city } \\
\text { use case with heterogene- } \\
\text { ous devices, algorithms, } \\
\text { protocols, and network } \\
\text { configurations }\end{array}$ & smart city WSNs & 85 & $5 \%-10 \%$ \\
\hline SVM and RBF and [23] & $\begin{array}{l}\text { SVM using Radial-basis } \\
\text { kernel function (RBF) and a } \\
\text { Particle Swarm Optimization } \\
\text { algorithm to optimize the } \\
\text { parameters of SVM }\end{array}$ & NSL-KDD & 99.8 & 0.9 \\
\hline SVM and GA [24] & $\begin{array}{l}\text { Hybrid classification algorithm } \\
\text { (GSVM) based Gravitational } \\
\text { Search Algorithm (GSA) and } \\
\text { support vector machines } \\
\text { (SVM) to optimize the accu- } \\
\text { racy of the SVM classifier by } \\
\text { detecting the subset of the } \\
\text { best values of the kernel } \\
\text { parameters for the SVM } \\
\text { classifier }\end{array}$ & KDD CUP 99 & 97.5 & 0.03 \\
\hline $\begin{array}{l}\text { Support Vector Machine (SVM) } \\
\text { Based on Wavelet Transform } \\
\text { (WT) [25] }\end{array}$ & $\begin{array}{l}\text { Support Vector Machine } \\
\text { SVM based on Wavelet } \\
\text { Transform(WT) }\end{array}$ & UNSW-NB15 & 95.92 & - \\
\hline
\end{tabular}

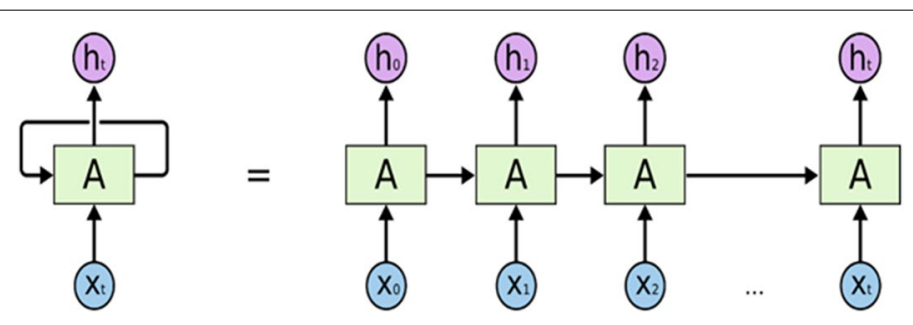

Fig. 2 RNN have Loops [27]. Shows simple diagram of recurrent neural networks

where $x t, \mathrm{~h} t$, and $c t$ correspond to input layer, hidden layer, and cell state at time $t$. Besides, $b i, b f, b c$, and $b o$ are bias at input gate, forget gate, cell state, and output gate, respectively. Furthermore, $\sigma$ is sigmoid function. Finally, $W$ is denoted by weight matrix 


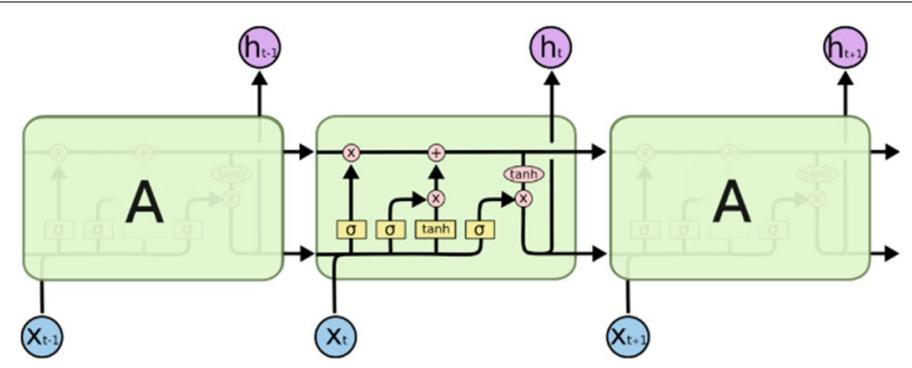

The repeating module in an LSTM contains four interacting layers.

Fig. 3 LSTM architecture [39]. Shows in details architecture of long short term memory
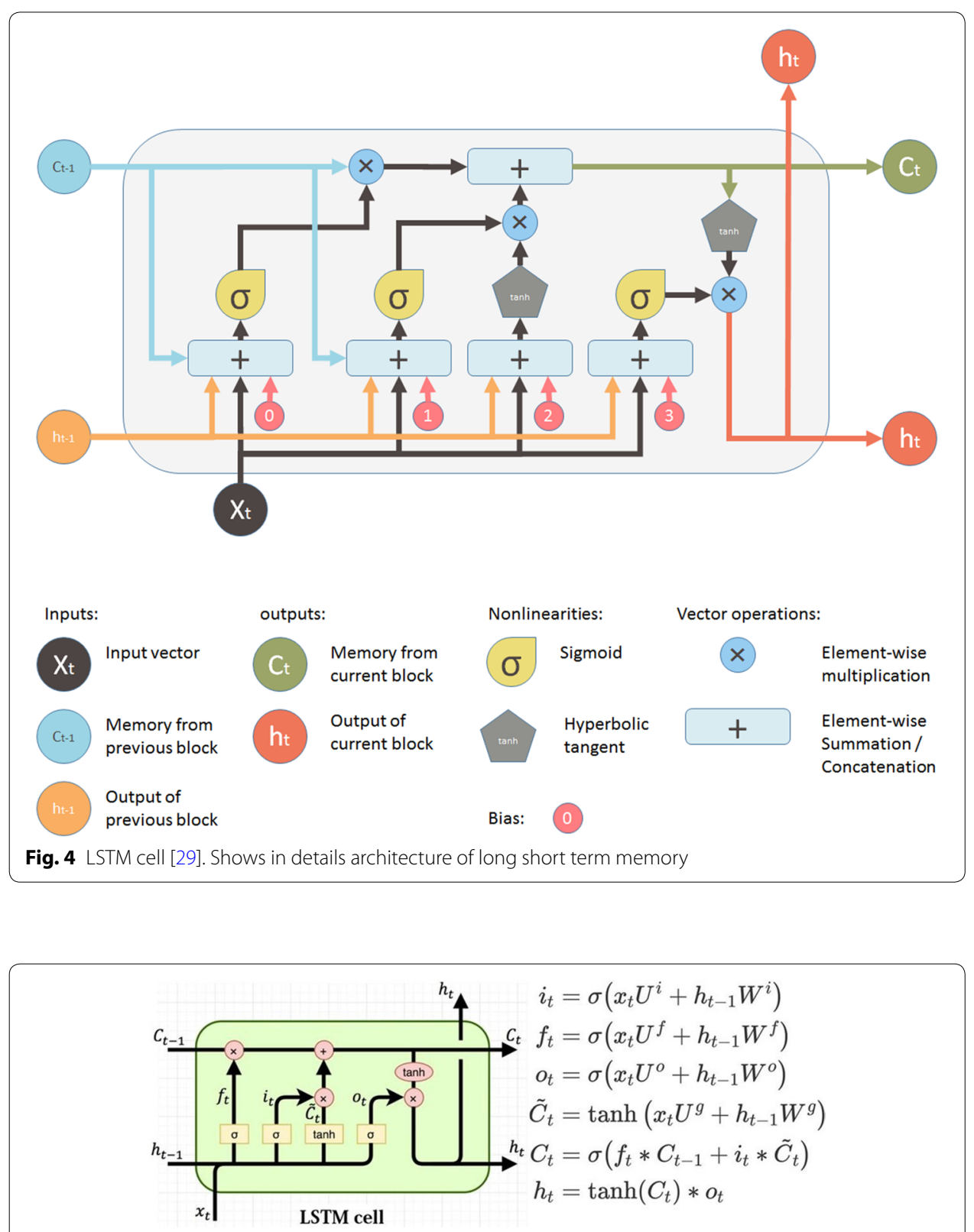

Fig. 5 LSTM cell equations [38]. Shows in details equations of LSTM Cell 
Table 2 Statistics about KDD training set [33]

\begin{tabular}{llll}
\hline & Number of samples & Number of distinct samples & $\begin{array}{l}\text { Possible reduction } \\
\text { percentage }\end{array}$ \\
\hline Attacks & $3,925,650$ & 262,178 & $93.32 \%$ \\
Normal & 972,781 & 812,814 & $16.44 \%$ \\
Total & $4,898,431$ & $1,074,992$ & $78.05 \%$ \\
\hline
\end{tabular}

Table 3 Statistics about KDD testing set [33]

\begin{tabular}{llll}
\hline & Number of samples & Number of distinct samples & $\begin{array}{l}\text { Possible reduction } \\
\text { percentage }\end{array}$ \\
\hline Attacks & 250,436 & 29,378 & $88.26 \%$ \\
Normal & 60,591 & 47,911 & $20.92 \%$ \\
Total & 311,027 & 77,289 & $75.15 \%$ \\
\hline
\end{tabular}

\section{Experiment setup}

This experiment is performed on Google CoLab [30] using Keras libary (Python Deep Learning library) [31]. We apply LSTM of 64 hidden nodes with Relu activation function and dropout $=0.5$. Using binary cross-entropy loss function. Using RMSprop optimizer. Learning rate $=0.001$, rho $=0.9$, decay $=0.0$.

\section{Data}

KDD99

This dataset is an updated version of the DARPA98 by processing the tcpdump portion. it was constructed in 1999 by the international competition, International Knowledge Discovery and Data Mining Tools Competition. Its size is $708 \mathrm{MB}$ and it contains about 5 million connections [32]. It contains different attacks such as Neptune-DoS, pod-DoS, SmurfDoS, and buffer-overflow. The benign and attack traffic are merged together in a simulated environment but it contains a large number of redundant records [33]

KDD99 is the most famous research dataset but it cannot be used for real-life applications as data is old, not real, not enough. Statistics details are provided in Table 2 and Table 3.

\section{Statistics about KDD training set}

\section{Statistics about KDD testing set}

\section{NSL-KDD}

This dataset is available online on website of Canadian Research for Intrusion detection [34]

NSL-KDD is built by resampling KDD99 to solve all its drawbacks that reduce the precision of mining algorithms on it. Resampling is done by removing duplicated samples and redundant samples. As KDD training set has $78 \%$ of duplicated samples and testing 


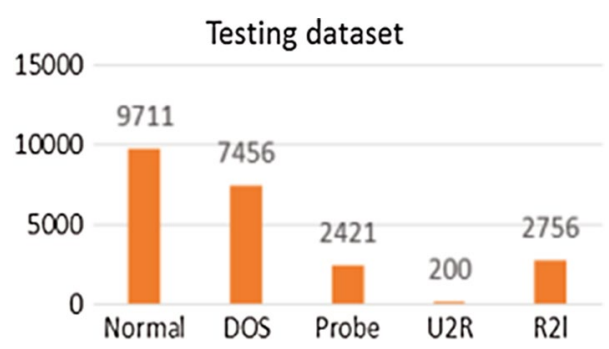

Fig. 6 Statistics about NSL-KDD testing dataset [3]. Shows Statistics about NSL-KDD training datasets. Statistics about number of samples for each type of attack

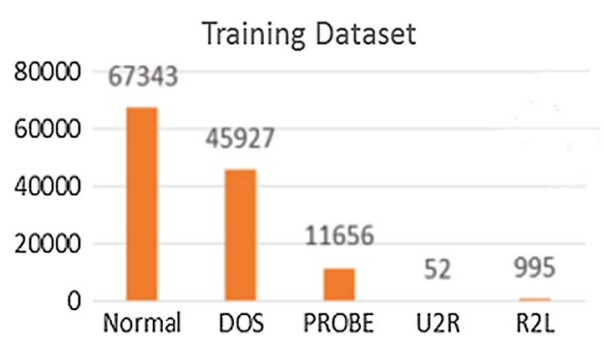

Fig. 7 Statistics about NSL-KDD training dataset [3]. Shows Statistics about NSL-KDD testing datasets. Statistics about number of samples for each type of attack

set has $75 \%$ of duplicated samples that result to bias detection to detect attacks that have more number of samples and make it hard to learn attacks that have small number of samples but may be one most dangerous attacks, such as U2R, L2R.

\section{NSL_KDD advantages}

- It does not contain redundant samples in testing samples so that solves bias problem

- It does not contain duplicate records in the test set which have better reduction rates.

- All samples for any attack type has the same percentage of its number in KDD.

- It contains 21 attacks in training dataset and 37 attacks in testing dataset so we can test the unknown attacks detection capability of a model by testing on attacks that some of them are new for the model as they were not in training set $[3,33]$.

\section{NSL statistics}

Statistics about NSL_KDD training and testing datasets in Figs. 6, 7.

Where $\mathrm{x}$-axes represent attacks types, $\mathrm{y}$-axes represent number of samples.

NSL-KDD is not biased but that does not mean that it is optimal. It has many drawbacks, but still more effective than other available datasets. At least, it will become out-of-date after few months as developing technologies and new attacks appear every day. 
Table 4 Type of attacks available in NSL-KDD [3]

\begin{tabular}{ll}
\hline Attack type & Attack name \\
\hline DoS & Back, Land, Neptune, Pod, Smurf, Teardrop, Mailbomb, Pro- \\
R2L & cestable, Udpstorm, Apache2, Worm \\
& Guess_password, Ftp_write, Imap, Phf, Multihop, Warezmaster, \\
& Xlock, Xsnoop, Snmpguess, Snmpgetattack, Httptunnel, \\
& Sendmail, Named \\
U2R & Buffer_overflow, Loadmodule, Rootkit, Perl, Sqlattack, Xterm, Ps \\
Probe & Satan, IPsweep, Nmap, Portsweep, Mscan, Saint \\
Normal & Normal \\
Unknown & Unknown \\
\hline
\end{tabular}

\begin{tabular}{|cccccc|}
\hline F\# & Feature name & F\# & Feature name & F\# & Feature name \\
\hline F1 & Duration & F15 & Su attempted & F29 & Same srv rate \\
F2 & Protocol type & F16 & Num root & F30 & Diff srv rate \\
F3 & Service & F17 & Num file creations & F31 & Srv diff host rate \\
F4 & Flag & F18 & Num shells & F32 & Dst host count \\
F5 & Source bytes & F19 & Num access files & F33 & Dst host srv count \\
F6 & Destination bytes & F20 & Num outbound cmds & F34 & Dst host same srv rate \\
F7 & Land & F21 & Is host login & F35 & Dst host diff srv rate \\
F8 & Wrong fragment & F22 & Is guest login & F36 & Dst host same src port rate \\
F9 & Urgent & F23 & Count & F37 & Dst host srv diff host rate \\
F10 & Hot & F24 & Srv count & F38 & Dst host serror rate \\
F11 & Number failed logins & F25 & Serror rate & F39 & Dst host srv serror rate \\
F12 & Logged in & F26 & Srv serror rate & F40 & Dst host rerror rate \\
F13 & Num compromised & F27 & Rerror rate & F41 & Dst host srv rerror rate \\
F14 & Root shell & F28 & Srv rerror rate & F42 & Class label \\
Fig. 8 & NSL KDD features [34]. Shows features of NSL-KDD dataset & & \\
\hline \multicolumn{7}{r}{} & & &
\end{tabular}

\section{Type of attacks available in NSL-KDD}

NSL-KDD contains 37 types of attacks that are categorized into 6 basic categories.

Categories are DoS, R2L, U2R, Probe, Normal, Unknown. Find details in Table 4.

\section{NSL-KDD attributes}

NSL-KDD contains 41 attributes as we can find in Fig. 8 [35].

\section{Results and discussion}

We will not discuss that big data is better with deep learning than traditional learning. It is a fact that the most important difference between deep learning and traditional machine learning is its performance as the scale of data increases. As we can see in Fig. 9 [36].

We did not use a big dataset in this experiment. We used a small dataset as we want to compare the results of applying deep learning instead of traditional learning in anomaly-based IDS even on small dataset. Thus, we can compare optimization of generalization. We got by experiment, that accuracy increases by using deep learning 


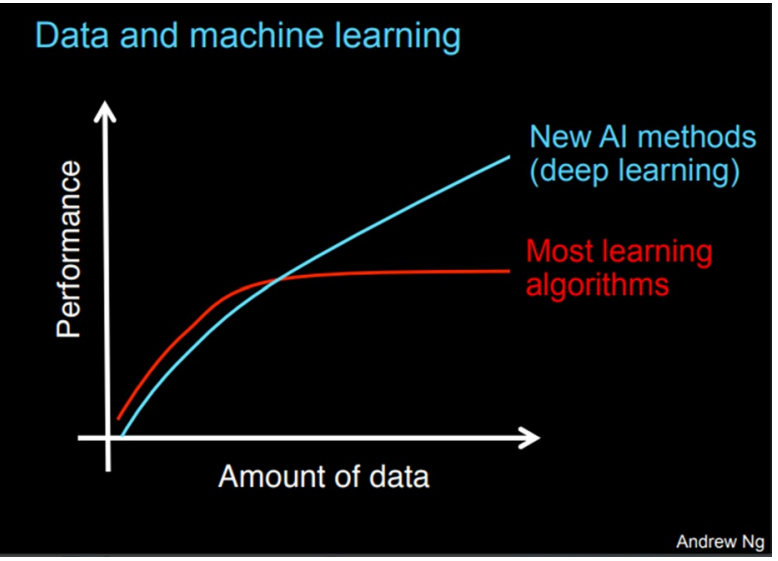

Fig. 9 Big data with deep learning performance optimization [36, 37]. Shows performance optimization when using bigger amount of data

Table 5 Comparing false-positive of Deep vs SVM on NSL-KDD Benchmark

\begin{tabular}{llll}
\hline Methodology & False-positive FP & False negative FN & Accuracy \\
\hline Deep learning (LSTM) & 0.01 & 0.03 & 0.9676 \\
SVM & 0.1 & 0.03 & 0.87 \\
\hline
\end{tabular}

model instead of traditional learning model. Moreover, false positive is getting lower by $10 \%$ less than traditional learning models. Results are shown in Table 5.

We used SVM to compare with, because it has one of the highest results among traditional learning classifiers. The result we get is higher than all previous studies we have shown in related works.

\section{Conclusion and future work}

Using big data analysis with deep learning in anomaly detection shows excellent combination that may be optimal solution as deep learning needs millions of samples in dataset and that what big data handle and what we need to construct big model of normal behavior that reduce false-positive rate to be better than small traditional anomaly models.

We recommend using deep learning models instead of SVM when trying to build hybrid classifiers for IDS. Because, as we see in results, deep models have more ability to generalize than traditional models. Thus, deep models achieve better results on unseen data causing less false-positive rate. We recommend using LSTM as we think it is an optimal solution in security domain because it can deal with sequences of events and context. So that it cannot only achieve better accuracy, but also can detect more various types of attacks that were not possible before. Such as, contextual and collective attacks.

\section{Abbreviations}

IDS: Intrusion detection system; RNN: Recurrent neural networks; LSTM: Long short term memory; FP: False-positive; DoS: Deny of service attack; R2L: Remote to user; U2R: User to root. 


\section{Acknowledgements}

I would like to thank my parents for their endless love and support.

\section{Authors' contributions}

$\mathrm{KHJ}$ took on the main role so she performed the literature review, conducted the experiments and wrote the manuscript. $\mathrm{MJ}$ and SD took on a supervisory role and oversaw the completion of the work. All authors read and approved the final manuscript.

Funding

The authors declare that they have no funding.

Availability of data and materials

All datasets in this survey are available online, you can find links in references.

Ethics approval and consent to participate

The authors Ethics approval and consent to participate.

\section{Consent for publication}

The authors consent for publication.

\section{Competing interests}

The authors declare that they have no competing interests.

\section{Author details}

${ }^{1}$ Faculty of Information Technology, Higher Institute for Applied Sciences and Technology, Damascus, Syria. ${ }^{2}$ Faculty of Information Technology, Arab International University, Daraa, Syria.

Received: 28 March 2019 Accepted: 14 August 2020

Published online: 31 August 2020

\section{References}

1. Bijone M. A survey on secure network: intrusion detection \& prevention approaches. Am J Inf Syst. 2016;4(3):69-88.

2. Calix RA, Sankaran R. Feature ranking and support vector machines classification analysis of the NSL-KDD intrusion detection corpus. In: FLAIRS conference. 2013. https://www.semanticscholar.org/paper/Feature-Ranking-andSupport-Vector-Machines-of-the-Calix-Sankaran/dfd45d96fc8ddb366ca109ec62dfbf8c9f56f842

3. Revathi S, Malathi DA. A detailed analysis on NSL-KDD dataset using various machine learning techniques for intrusion detection. Int J Eng Res Technol. 2013;2(12):1848-53.

4. Suthaharan S. Big data classification: problems and challenges in network intrusion prediction with machine learning. Perform Eval Rev. 2014;41(4):70-3.

5. Tao P, Sun Z, Sun Z. An improved intrusion detection algorithm based on GA and SVM. IEEE Access. 2018;6:13624-31.

6. SVM_figure, researchgate, [Online]. 2019. https://www.researchgate.net/figure/Classification-of-data-by-suppo rt-vector-machine-SVM_fig8_304611323. Accessed 2019.

7. Song J, Takakura H, Okabe Y, Nakao K. Toward a more practical unsupervised anomaly detection system. Inf Sci. 2013;231:4-14.

8. Zhao G, Song J, Song J. Analysis about performance of multiclass SVM applying in IDS. In: Proceedings of the 2013 International Conference on Information, Business and Education Technology (ICIBET 2013). Atlantis Press; 2013. https://www.researchgate.net/publication/266648815_Analysis_about_Performance_of_Multiclass_SVM_Apply ing_in_IDS

9. Araki S, Yamaguchi Y, Shimada H, Takakura H. Unknown attack detection by multistage one-class SVM focusing on communication interval. In: Loo CK, Yap KS, Wong KW, Beng Jin AT, Huang K (eds) Neural information processing. ICONIP 2014. Lecture notes in computer science, vol 8836. Cham: Springer; 2014. https://doi.org/10.1007/978-3319-12643-2_40.

10. Enache A, Patriciu V. Intrusions detection based on support vector machine optimized with swarm intelligence. In: 2014 IEEE 9th IEEE international symposium on applied computational intelligence and informatics (SACI). Timisoara, Romania: IEEE; 2014. p. 153-158. https://doi.org/10.1109/SACl.2014.6840052

11. Catania CA, Bromberg F, Garino CG. An autonomous labeling approach to support vector machines algorithms for network traffic anomaly detection. Expert Syst Appl. 2012;39(2):1822-9.

12. Yi Y, Wu J, Xu W. Incremental SVM based on reserved set for network intrusion detection. Expert Syst Appl. 2011;38(6):7698-707.

13. K. Atefi, S. Yahya, A. Y. Dak, A. Atefi, A hybrid intrusion detection system based on different machine learning algorithms. In: 4th International Conference on Computing and Informatics, Sarawak, Malaysia, 2013.

14. Ahmad I, Hussain M, Alghamdi A, Alelaiwi A. Enhancing SVM performance in intrusion detection using optimal feature subset selection based on genetic principal components. Neural Comput Appl. 2014;24(7-8):1671-82.

15. Sung AH, Mukkamala S. Identifying important features for intrusion detection using support vector machines and neural networks. In: 2003 symposium on applications and the internet, 2003. Proceedings. Orlando, FL, USA: IEEE; 2003, p. 209-216. https://doi.org/10.1109/SAINT.2003.1183050.

16. Kuang F, Xu W, Zhang S. A novel hybrid KPCA and SVM with GA model for intrusion detection. Appl Soft Comput. 2014;18:178-84.

17. Chitrakar R, Huang C. Selection of candidate support vectors in incremental SVM for network intrusion detection. Comput Secur. 2014;45:231-41. 
18. Khalvati L, Keshtgary M, Rikhtegar N. Intrusion detection based on a novel hybrid learning approach. JAIDM. 2017:6(1):157-62.

19. Guo C, Zhou Y, Ping Y, Zhang Z, Liu G, Yang Y. A distance sum-based hybrid method for intrusion detection. Appl Intell. 2014;40(1):178-88.

20. Aslahi-Shahri B, Rahmani R, Chizari M, Maralani A, Eslami M, Golkar M, et al. A hybrid method consisting of GA and SVM for intrusion detection system. Neural Comput Appl. 2015;27(6):1669-766.

21. Ikram ST, Cherukuri AK. Improving accuracy of intrusion detection model using PCA and optimized SVM. J Comput Inf Technol. 2016;24(2):133-48.

22. Garcia-Font V, Garrigues C, Rifà-Pous H. Difficulties and challenges of anomaly detection in smart cities: a laboratory analysis. Sensors. 2018;18(10):3198.

23. Chakir EM, Moughit M, Khamlichi YI. An effective intrusion detection model based on SVM with feature selection and parameters optimization. J Theor Appl Inf Technol. 2018;96(12):3873-85.

24. Benqdara S. Anomaly intrusion detection based on a hybrid classification algorithm (GSVM). Int J Comp Appl. 2019;181(36):0975-8887.

25. Kurnaz S, Obaid IA. Support vector machine (SVM) based on wavelet transform (WT) for intrusion detection system (IDS). Int J Comp Sci Mob Comput. 2019;8(2):13-9.

26. Jallad KA, Aljnidi M, Desouki MS. Big data analysis and distributed deep learning for next-generation intrusion detection system optimization. J Big Data. 2019;6:88.

27. "Understanding-LSTMs," [Online]. 2015. https://colah.github.io/posts/2015-08-Understanding-LSTMs/. Accessed Aug 2015.

28. Hochreiter S, Schmidhuber J. Long short-term memory. Neural Comput. 1997;9(8):1735-80

29. "Understanding-Istm-and-its-diagrams," medium, [Online]. 2019. https://medium.com/mlreview/understandingIstm-and-its-diagrams-37e2f46f1714. Accessed 2019.

30. "Colab," Google, [Online]. 2018. https://colab.research.google.com/. Accessed 2018.

31. "Keras," [Online]. 2018. https://keras.io/. Accessed 2018.

32. "KDD Cup 1999 Data" [Online]. 1999. https://kdd.ics.uci.edu/databases/kddcup99/kddcup99.html. Accessed 2017.

33. Tavallaee M, Bagheri E, Lu W, Ghorbani AA. A detailed analysis of the KDD CUP 99 data set. In: 2009 IEEE symposium on computational intelligence for security and defense applications, Ottawa, ON: IEEE; 2009. p. 1-6. https://doi. org/10.1109/CISDA.2009.5356528.

34. "NSL-KDD," [Online]. https://www.unb.ca/cic/datasets/nsl.html. Accessed 10 Oct 2019.

35. "List-of-features-of-NSL-KDD-dataset," Researchgate [Online]. 2019. https://www.researchgate.net/figure/List-offeatures-of-NSL-KDD-dataset_tbl1_325709588. Accessed 2019.

36. Andrew Ng. Machine learning yearning, deeplearning.ai project, draft version. Andrew Ng; 2018. https://www.deepl earning.ai/machine-learning-yearning/.

37. A. NG. Deep learning course. http://cs229.stanford.edu/materials/CS229-DeepLearning.pdf. Accessed 10 Oct 2019

38. "ResearchGate," [Online]. https://www.researchgate.net/figure/Structure-of-the-LSTM-cell-and-equations-that-descr ibe-the-gates-of-an-LSTM-cell_fig5_329362532. Accessed 10 Oct 2019.

39. Understanding-architecture-of-Istm-cell-from-scratch-with-code. 2018. https://medium.com/m/signin?redir ect=https\%3A\%2F\%2Fhackernoon.com\%2Funderstanding-architecture-of-Istm-cell-from-scratch-with-code-8da40 fob71f4\%3Fsource\%3Dquote menu. Accessed 10 Oct 2019.

\section{Publisher's Note}

Springer Nature remains neutral with regard to jurisdictional claims in published maps and institutional affiliations.

\section{Submit your manuscript to a SpringerOpen ${ }^{\circ}$ journal and benefit from:}

- Convenient online submission

Rigorous peer review

- Open access: articles freely available online

- High visibility within the field

- Retaining the copyright to your article

Submit your next manuscript at $\gg$ springeropen.com 